Archives de sciences sociales des religions

141 | janvier-mars 2008

Mémoires

\title{
Religion, violence et politique chez les Mayas du Chiapas
}

Jean-Pierre Bastian

\section{OpenEdition}

Journals

Édition électronique

URL : https://journals.openedition.org/assr/12812

DOI : 10.4000/assr. 12812

ISSN : $1777-5825$

Éditeur

Éditions de l'EHESS

Édition imprimée

Date de publication : 1 mars 2008

ISBN : 978-2-7132-2189-7

ISSN : 0335-5985

\section{Référence électronique}

Jean-Pierre Bastian, «Religion, violence et politique chez les Mayas du Chiapas », Archives de sciences sociales des religions [En ligne], 141 | janvier-mars 2008, mis en ligne le 15 juin 2011, consulté le 05 juillet 2021. URL : http://journals.openedition.org/assr/12812 ; DOI : https://doi.org/10.4000/assr. 12812

(C) Archives de sciences sociales des religions 


\section{Jean-Pierre Bastian}

\section{Religion, violence et politique chez les Mayas du Chiapas}

Le soulèvement de l'armée zapatiste de libération nationale (EZLN) dans l'État du Chiapas (janvier 1994) a alerté l'opinion publique internationale sur les graves problèmes sociaux affectant les communautés indiennes du sud du Mexique. Le Chiapas (plus de 3,2 millions d'habitants en 2000) possède une importante population ethnique avec $26 \%$ d'indiens mayas (Tzeltales, Tzotziles, Choles, Tojolobales, Mames, Chujes et Kanjobales) et zoques. Héritier d'une des principales cultures amérindiennes, cet État occupe le premier rang au sein de la Fédération mexicaine pour son taux d'analphabétisme : $30 \%$ de la population âgée de plus de quinze ans, $60 \%$ dans les régions indiennes. Les contrastes économiques y sont saisissants. En dépit d'une grande richesse en ressources naturelles (pétrole, agriculture tropicale, café), la marginalisation et l'exclusion prédominent, surtout parmi les populations indiennes.

Cette situation reflète des rapports sociaux de longue durée qui, dès le $\mathrm{XVI}^{\mathrm{e}}$ siècle, se sont fondés sur la coercition et sur l'asymétrie structurelle entre les « villes d'Espagnols » et les « villages d'Indiens ». Les mouvements récurrents de protestation indienne (Barabas, 1989) en furent la conséquence. Cependant, depuis une trentaine d'années, la violence affecte les relations intra-ethniques elles-mêmes. Deux facteurs se conjuguent pour acculer les populations indigènes à la violence : les caciques indiens, tout autant que les grands propriétaires métis, accaparent et accumulent les meilleures terres; la croissance démographique accélérée redouble la pression sur la terre et pousse les jeunes à chercher des emplois en dehors de leur communauté ou à survivre dans des conditions d'extrême précarité. L'apparition du mouvement armé zapatiste s'inscrit dans ce contexte. Parmi les indicateurs de la révolte, le phénomène religieux de l'expansion des sectes protestantes, en particulier en territoire indien, est sans doute un révélateur de cette " rébellion annoncée » et des changements en cours au sein des communautés indiennes. Je voudrais montrer comment la différenciation religieuse, expression et acteur des conflits sociaux, est un moyen de construire un nouveau rituel qui vise à briser l'ancien rituel politico-religieux communautaire, instrument principal de la coercition politique intra-ethnique, dans une région où s'est structurée depuis 
la période coloniale une société raciste, de "castes » opposant indiens, métis et blancs. L'adoption de nouvelles ritualités par la multiplication d'entreprises religieuses en concurrence affecte, certes, la sociabilité ethnique. En centrant le conflit intra-ethnique autour de valeurs morales, les dissidents protestants mettent en question le politique légitime imposé par l'État via les affiliations partisanes. Ils construisent un conflit politico-religieux dont ils sont les régulateurs, ce que le politique légitime des organisations partisanes nationales ne leur permettait pas d'assurer.

\section{Une mutation des affiliations religieuses}

Les recensements décennaux de l'Institut mexicain des statistiques (INEGI, Instituto Nacional de Estadística, Geografía e Informática) montrent l'évolution progressive du pays vers une pluralité religieuse relative, mais de plus en plus affirmée sur ses marges frontalières. En 2000, 87 \% des mexicains se disaient catholiques et les protestants atteignaient un peu plus de $7 \%$ de la population avec une faible présence dans le centre-ouest du pays (Bajío), densément peuplé où prédomine un catholicisme institutionnel de longue implantation. En revanche, le phénomène de pluralisation des appartenances religieuses a pris une ampleur considérable dans les régions périphériques, en particulier dans les États « indiens » du sud du pays (Tabasco, Oaxaca, Yucatan, Quintana Roo, Chiapas).

Dans l'État du Chiapas, le mouvement s'est exacerbé au cours de ces vingtcinq dernières années. Alors qu'en 1970, $91 \%$ des habitants se définissaient «catholiques ", ils n'étaient plus que $76 \%$ à le faire en 1980, $67 \%$ en 1990 et $63 \%$ en 2000, selon les recensements de l'INEGI. Aujourd'hui, le Chiapas est l'État du Mexique au taux le plus élevé de non-catholiques romains; ceux-ci appartiennent parfois, dans certains municipes (territoires communaux), au catholicisme coutumier ; cependant, la plupart des non-catholiques sont membres d'organisations religieuses relevant surtout du protestantisme historique (presbytérien, baptiste, nazaréen), du pentecôtisme (Assemblées de Dieu, Église de Dieu de la Prophétie) et de mouvements religieux parachrétiens divers (Témoins de Jéhovah, Luz del Mundo, Mormons, Adventisme). Pour la première fois en cinq siècles de présence, l'Église catholique, avec ses trois diocèses (San Cristobal, Tuxtla Gutiérrez et Tapachula) ne parvient plus à réguler les croyances de la population. Alors que, jusque-là, les expressions religieuses indiennes recouvertes par le terme de "coutume " s'étaient manifestées en rapport de subordination à l'autorité épiscopale reconnue, aujourd'hui, le catholicisme coutumier n'hésite pas à s'opposer au catholicisme institutionnel, surtout lorsque ce dernier développe des pratiques ecclésiales proches du courant de la théologie de la libération, comme ce fut le cas dans le diocèse de San Cristobal de las Casas. Par ailleurs, des pans entiers de la population adoptent des pratiques religieuses nouvelles, protestantes ou non, et échappent ainsi à la régulation du catholicisme institutionnel. 
Une sociographie détaillée révèle un processus qui, à court terme, peut amener l'Église catholique à se voir dépossédée de son monopole sur les consciences et à perdre même son hégémonie en territoire ethnique. Selon les données recueillies en 2000 par l'INEGI, ceci se produit déjà dans trente-trois des cent onze municipes (grandes communes) de l'État (alors que ce n'était le cas que dans vingt-cinq d'entre eux en 1990) où les catholiques représentent moins de $50 \%$ de la population. Cette géographie de la décatholicisation coïncide avec celle des périphéries ethniques, avec les régions où la violence agraire est la plus forte et où la guérilla zapatiste s'est implantée. Elle prédomine dans trois régions à dominance indienne : les Altos de Chiapas (Hauts du Chiapas), les Cañadas et la zone Fronteriza (Mariscal). Dans les Altos de Chiapas, certains municipes indigènes étaient déjà, en 1990, en majorité non catholiques romains ou en passe de le devenir et cette tendance s'est accélérée ${ }^{1}$. Dans d'autres municipes, le monolithisme catholique apparent à San Juan Chamula $74 \%$ (70,4 \% en 1990) et à Zinacantan 90,6 \% $(94,2 \%)$ est dû à l'expulsion des familles hétérodoxes par les caciques indiens au pouvoir. Dans ces deux derniers municipes, au cours de ces trente dernières années, des milliers d'habitants ont été chassés par la force vers les périphéries de la capitale régionale, San Cristobal de las Casas, ou ont émigré vers la région pionnière des Cañadas. Dans cette deuxième région, aujourd'hui en partie territoire zapatiste, ils ont constitué des hameaux entièrement "protestants ", changeant ainsi la composition religieuse des municipes d'accueil comme à La Independencia où, en 2000, il n'y avait plus que 33,2 \% de catholiques, à Las Margaritas 62,2 \% et à Ocosingo 46,2 \%. La tendance est encore plus affirmée dans la zone Fronteriza où prédominent les communautés indiennes Mams ${ }^{2}$. Au cours des années 1980, bouleversées par l'afflux d'indiens mams guatémaltèques chassés par l'affrontement entre troupes gouvernementales et guérilla, les communautés mams ont expérimenté, de part et d'autre de la frontière (García Ruiz, 1988), une véritable mutation des affiliations religieuses qui semble restée stable aux regards des données statistiques de 1990 et de 2000.

Le Chiapas a toujours été une mosaïque ethnique. Les ethnies actuelles sont le fruit de reconstructions identitaires de longue durée qui suivirent l'effondrement démographique lié à la conquête espagnole. L'État colonial se servit des divisions ethniques reconstituées afin de faciliter le contrôle politique de la région. Bien que des mouvements messianiques indiens aient éclaté de manière sporadique (Favre, 1971), il n'y eut jamais d'Indiens unis dans un projet commun de résistance à l'occupant. En revanche, jusqu'aux années 1950, une certaine stabilité dans la

1. Puisque les catholiques ne sont que $33,4 \%$ à Chanal (alors qu'ils étaient encore $49,2 \%$ en 1990$)$, à Chenalho $16,7 \%(43,4 \%)$, à Oxchuc $47,4 \%(52,8 \%)$, à Mitontic $46,5 \%$ $(92,8 \%)$ et à Tenejapa $35,8 \%(37,4 \%)$.

2. En 2000, les catholiques romains ne constituaient plus que $17,8 \%(20 \%)$ de la population du municipe de Bejucal, $29 \%$ (27\%) de celui d'El Porvenir, 28,7\% (27,8\%) de Bella Vista, $39 \%$ (35 \%) de La Grandeza, 39,5 \% (40 \%) de Motozintla, $42 \%$ (43,7 \%) de Mazapa, $43 \%$ $(48 \%)$ de Frontera Comalapa, $54 \%(52 \%)$ de Amatenango et $61 \%(59,4 \%)$ de Siltepec. 
répartition territoriale et un même univers symbolique civico-religieux assurèrent une grande homogénéité aux identités ethniques. Depuis lors, les expulsions et les flux migratoires frontaliers ont renforcé l'atomisation des populations indiennes en particulier dans les régions pionnières où des hameaux ethniques se succèdent sans autre logique que celle de la migration de groupes de parenté partageant une même identité ethnique. À cela, se sont ajoutées les tensions intra-ethniques liées à une différenciation sociale accélérée par l'expansion de l'économie de marché et les affrontements entre indiens, et entre indiens et métis autour du problème agraire. La multiplication des appartenances religieuses est le fruit de ce processus qu'elle renforce. Il nous faut expliquer pourquoi et comment.

\section{Guerres de religion et reproduction ethnique}

Dans les communautés indiennes, le religieux et le politique ont toujours été mêlés et la ritualité religieuse est un facteur clé de la reproduction sociale. L'anthropologie fonctionnaliste a montré de quelle manière le système des fêtes repose sur une organisation religieuse, les confréries et les majordomies. Il s'agit d'associations dont les membres se recrutent parmi certains métiers ou parmi certains quartiers des municipes (Lundius, 1993). À tour de rôle, les individus représentant leur famille étendue sont chargés d'assurer les rites constitutifs de la fête religieuse cyclique. De là, le terme de "charge " qui a été retenu par le caractère coercitif de la fonction qui engage temps et argent. Le système rituel des charges fait entrer l'individu dans une hiérarchie et structure ainsi la communauté ethnique. En même temps, il assure une mobilité symbolique orientée vers la recherche de prestige liée à l'exécution de la charge. Pour de nombreux anthropologues, le système rituel des fêtes religieuses a été la base et la cause de la stabilité sociale et culturelle indienne. La participation aux charges assure l'intégration communautaire tout autant que le nivellement économique de la société indienne. En effet, dans l'économie traditionnelle, pré-capitaliste, les dépenses rituelles, somptuaires, assumées à tour de rôle par les familles érodaient la richesse des Indiens les plus prospères et les empêchaient de se transformer en entrepreneurs. De cette manière la communauté indigène se nivelait au travers de ce processus de consumation de l'excédent empêchant toute mobilité sociale autre que symbolique et prévenait les initiatives économiques capables de défaire le lien communautaire (Cancian, 1965).

Cette analyse présuppose la stabilité des entités ethniques et a pu refléter une certaine réalité des sociétés indiennes, mais elle a conduit, sur le plan théorique, à la substantialisation de l'identité ethnique ${ }^{3}$. Elle s'est trouvée mise à mal par la brusque insertion des sociétés indiennes à l'économie de marché par le biais des politiques de développement promues par l'État mexicain au cours des années 1940 et 1950. Ceci a provoqué chez les Tzotzils et les Tzeltals des Altos de

3. Pour une discussion du concept d'ethnicité, voir Poutignat et alii, 1995, pp. 134-153. 
Chiapas, l'accroissement des différences sociales intra-ethniques. Comme l'ont constaté Smith (1981) et Cancian (1992), aujourd'hui le système rituel des fêtes n'a aucun effet sur la redistribution de l'excédent. Les fêtes ne font qu'intensifier la consommation. Elles ont généré un marché lucratif lié au rituel religieux. La vente d'alcool (de posh pour être plus précis), de bougies, de fleurs et plus récemment de boissons gazéifiées procure des revenus conséquents à ceux qui servent d'intermédiaires commerciaux, détiennent le transport et le commerce et se donnent ainsi les moyens financiers d'accaparer la terre. Si l'on tient compte du fait que les communautés indiennes célèbrent en moyenne une fête religieuse par mois ${ }^{4}$ et que les Indiens ont constamment recours aux faveurs des saints de leur choix, on saisit l'importance du contrôle du marché rituel. Ceci renforce une stratification sociale asymétrique dans la mesure où ce sont les Indiens détenteurs des commerces et des moyens de transport et leurs «clients politiques " qui assurent les fêtes religieuses, moments de consommation accrue d'alcool, de cierges, de nourriture et par là, d'endettement. En cela, les cérémonies rituelles ont été détournées de leur fonction redistributive; elles récompensent les Indiens les plus riches, les caciques, en leur offrant un prestige accru et en leur assurant le renforcement de leurs monopoles; elles servent à sanctionner et à légitimer les inégalités croissantes au sein même des communautés indiennes.

Pour comprendre ce détournement, il convient de rappeler que le caciquat, comme mode d'exercice du pouvoir dans les communautés indiennes, fut une création de l'État colonial. Il permit de maintenir un "système de royauté sacrée » (Dehouve, 2006 : 130-131), lié à un groupe de gouvernants exécutant "périodiquement des rituels au nom de l'ensemble de la communauté, dont une partie sont pénitenciaires ». Autour de ce noyau central de caciques se déploya la constellation des groupes périphériques au sein des municipes et en particulier les organisations rituelles appelées mayordomias. L'État révolutionnaire mexicain reprit à son compte le contrôle des populations indiennes au travers de ce système de médiation entre la société globale et les communautés indiennes qu'est le caciquat. L'État contribua à la formation d'une élite indienne associée aux politiques d'intégration suscitées par les appareils para-étatiques, en particulier l'Institut National Indigéniste. Dès les années 1920, les caciques reconnus ou imposés par l'État se sont rendu compte qu'une des manières de conserver le pouvoir consistait à maîtriser les ritualités politico-religieuses traditionnelles à leur profit. C'est ainsi que, forts de l'appui politique de l'État-parti révolutionnaire (PRI) dont ils disposaient, ils se sont servis de la fête rituelle pour renforcer un contrôle social qui s'impose, aujourd'hui comme hier, par le règne de l'arbitraire et par une violence intra-ethnique continue.

4. Par exemple, à San Juan Chamula les principales fêtes sont les suivantes: Sébastien Martyr (18-20 janvier); carnaval (février), Saint Joseph (19 mars), Semaine sainte (avril), Sainte Croix (mai), Saint Jean Baptiste (19-24 juin), Sainte Rose de Lima (28-30 août), Saint Matthieu l'apôtre (20-21 septembre), Vierge du Rosaire (5-7 octobre), Toussaint ( $1^{\text {er }}-2$ novembre), Noël (25 décembre). 
C'est précisément à partir du moment où s'est produit le détournement des ritualités religieuses au profit des caciques légitimés par le régime politique, que de nouveaux mouvements religieux et sectes (protestants, pentecôtistes et parachrétiens) se sont répandus dans les communautés indiennes. Ils sont une manifestation de la différenciation sociale croissante et des antagonismes sociaux intra-ethniques. L'adoption de nouvelles pratiques religieuses a concerné avant tout les secteurs indiens marginaux ou en voie de marginalisation. C'est pourquoi les Indiens protestants, pentecôtistes ou affiliés à divers autres nouveaux mouvements religieux, se trouvent de préférence dans les hameaux (parajes, ejidos) dispersés au sein des municipes, à la périphérie du chef-lieu, alors que les caciques détiennent les commerces et les maisons du centre et les meilleures terres. Cette géographie de l'adhésion aux nouveaux mouvements religieux souligne le fait que les conversions ne sont pas individuelles, mais suivent les réseaux de parenté au sein d'une même fraction du groupe ethnique comme l'a montré Siverts (1969) dans le contexte du municipe d'Oxchuc.

Les nouvelles pratiques cultuelles sont un moyen de faire la grève du système d'exploitation lié à la ritualité politico-religieuse et se traduisent en premier lieu par le refus de la participation au travail communautaire (tequio) gratuit dont bénéficient d'abord les caciques. En particulier, l'entretien des routes à bon compte est favorable à leurs entreprises puisqu'ils sont les propriétaires des camions et des bus. L'adoption de religions de type ascétique rejetant l'alcool, le tabac et les défoulements de la fête traditionnelle n'est pas un hasard. Il s'agit d'un choix délibéré visant l'autonomie symbolique par la grève de pratiques rituelles pour lesquelles l'ingérence d'alcool est incontournable. Les nouvelles pratiques religieuses ne se réduisent pas au refus de la consumation propre au rituel coutumier. Elles remettent aussi en cause le système des charges, de plus en plus coûteux et de moins en moins accessible aux jeunes générations du boom démographique. La tendance à la redéfinition du système des charges paraît générale. Dans certains villages, où les activités économiques se sont diversifiées et développées de manière notable, ces transformations ont conduit les chefs de famille des hameaux périphériques du municipe à chercher à éviter les dépenses excessives nécessaires à l'accès aux charges. Cette prise de distance s'est parfois concrétisée par l'érection de chapelles indépendantes visant à la création de systèmes de charges moins onéreux et relativement autonomes par rapport au centre du municipe (Smith, 1981 : 56-57). Dans ce cas, on peut parler d'une rupture ad intra qui ne met pas en péril le système et, en général, ne provoque pas de violence intra-ethnique. De même, dans d'autres villages hors du Chiapas, certains anthropologues ont pu observer l'abandon pur et simple du système des charges, ce qui ne conduit pas nécessairement au conflit, même si de nouveaux groupes religieux non catholiques prennent la relève symbolique (Dow, 1993). En revanche, dans les communautés indiennes où le système des charges est fortement structuré et actif, la construction de lieux de culte qui échappent au système symbolique des charges sape les mécanismes de contrôle du caciquat. Cela conduit à de violentes persécutions religieuses (emprisonnements, meurtres, 
incendies de temples et de domiciles, viols, lynchages, séquestres, expulsions...) de la part des caciques menacés dans leur hégémonie par la grève religieuse. Pour le Chiapas, entre 1960 et 2001, Rivera Farfan (2006: 141) enregistre trois cent trente-neuf conflits religieux dont $69 \%$ d'entre eux se sont produits entre 1991 et 2001, principalement dans la régions des Altos de Chiapas et dans la région Fronteriza. Dans cette dernière région, les conflits religieux se sont succédés en 1981, 1985 et 1987, puis ont été continus durant les années 1990, conduisant «les catholiques traditionalistes, dirigés par leurs représentants politiques et civils, à menacer, emprisonner, frapper, séquestrer, détruire les biens et expulser les familles qui professent une autre religion que la catholique ", sous le prétexte de la violation des valeurs communautaires (Rivera Farfan, 2006 : 278-279).

De même, dans les Altos de Chiapas, les caciques chamulas ont expulsé durant les années 1970 et 1980 quelques trente mille membres de leur municipe surpeuplé (près de cinquante mille habitants en 2000). L'adoption de pratiques presbytériennes, adventistes, puis pentecôtistes, par les secteurs sociaux marginaux chamulas à partir de 1964 s'est traduite par des affrontements pour le pouvoir municipal dès 1970. Dans un pays où la démocratie est une fiction, où elle s'exerce par le biais du clientélisme dont les relais vont du sommet de l'État aux bases indiennes, les caciques chamulas, soutenus par le Parti Révolutionnaire Institutionnel (PRI), dénoncèrent les effets d'acculturation des sectes. Par là, il cherchèrent à occulter le problème fondamental, celui de l'accumulation et de l'exercice du pouvoir au sein des communautés ethniques. De leur côté, les Indiens protestants chamulas, parfois alliés aux secteurs catholiques progressistes proches de l'évêque de San Cristobal, n'ont pas hésité à s'affilier à un parti de droite, le Parti d'Action Nationale, pour mener le combat (Gabbert 1999 : 361366). Au moment des élections municipales de 1974, alors que "protestants " et catholiques progressistes étaient parvenus à conduire au pouvoir un candidat d'opposition, les caciques ont procédé de manière arbitraire aux premières expulsions massives, alléguant le non respect des traditions. Malgré le droit constitutionnel à la liberté de culte, l'État n'a pas sanctionné ces actions illicites au prétexte de la destruction des identités ethniques par les sectes. Les caciques, tous affiliés au PRI, ont récupéré ainsi le pouvoir et ont maintenu le catholicisme coutumier. Mais, critiqués et sanctionnés par l'évêque progressiste de leur diocèse, ils n'ont pas hésité à rompre le lien privilégié au catholicisme romain et à s'affilier à une église orthodoxe schismatique dont le siège se trouve dans la capitale de l'État du Chiapas. Depuis, alors que la protestation religieuse n'a fait que s'accroître, les persécutions et expulsions n'ont plus cessé 5 . Dans d'autres municipes,

5. Les expulsions affectent la plupart des municipes indiens des Hauts du Chiapas et, en particulier, ceux de Chamula, Chalchihuitán, Chenalhó, Mitontic et Zinacantan, ainsi que de nombreux autres municipes dans la zone d'influence zapatiste, en particulier à Las Margaritas. Sur le cas de la communauté Chamula voir Canton Delgado, 1994, pp. 44-49 et Bastian, 1994, pp. 230-232. Voir aussi, Informe sobre el problema de las expulsiones en las comunidades indígenas de los Altos de Chiapas y los derechos humanos, Mexico, Comisión Nacional de Derechos Humanos, 1992, 37 p. et Proceso (Mexico), 907, 20 avril 1992, pp. 10-14. 
comme à Zinacantan, l'opposition aux caciques s'est appuyée sur un parti de gauche (le Parti de la Révolution Démocratique, PRD) face aux caciques affiliés au PRI ${ }^{6}$. De droite ou de gauche, le choix politique partisan en milieu indien est second par rapport aux options religieuses. La différenciation politique y est avant tout une différenciation religieuse. C'est pourquoi les conflits intra-ethniques apparaissent comme des guerres de religion. Dans un univers non sécularisé, c'est le religieux qui est porteur de rupture politique, et non les appareils politiques qui sont perçus comme des structures non indiennes. Ainsi, les indiens Mams du hameau de Las Ceibas, convertis en Témoins de Jéhovah, ont rejeté les modalités conventionnelles d'élection populaire, pour transformer l'élection en un service à la communauté défini pas les nouvelles autorités religieuses témoins de Jéhovah (Hernández Castillo, 2001 : 124).

Face aux agressions, les Indiens non catholiques ne sont pas restés passifs et ont parfois riposté avec les mêmes moyens que ceux utilisés par leurs adversaires. Mais de manière générale, les persécutions et les expulsions ont conduit plutôt les non-catholiques à s'organiser en associations de défense de leurs droits à la liberté de culte garantis constitutionnellement. Ces associations se convertirent en instances de représentation et de négociation vis-à-vis des autorités locales et nationales. Ainsi le "Comité de défense des menacés, persécutés et expulsés de Chamula "se transforma en Conseil de Représentants Indigènes des Altos de Chiapas (CRIACH) dès le début des années 1980. Au même moment, se créa l'Alliance Évangéliques des Ministres des Altos de Chiapas dont la tâche fut centrée sur des activités éducatives, d'action sociale et de coordination de campagnes d'évangélisation. Durant les années 1990, ce type d'association se développa dans le sens d'un front commun, en particulier à partir de l'expulsion massive d'évangéliques et de membres de sociétés para-chrétiennes dans divers municipes en 1993. Ainsi surgirent des centres de secours et de défense des droits humains tels les centres Fuente de Agua Viva (Source d'eau vive), Rescate de Dios (Sauvetage de Dieu) et le Centre de Droits Humains «Esteban » tous trois liés à l'Alliance Évangélique Ministérielle des Altos de Chiapas. De même, le centre Le Bon Samaritain, la Commission Évangélique des Droits Humains (CEDH) (liés à l'Église presbytérienne) et le Centre Vision de Aguila 2000 virent le jour. Dans la zone Fronteriza, l'Organisación de Pueblos Evangélicos Tojolobales (OPET) a été mise sur pied récemment (Rivera Farfan 2006 : 274 et 354). Ces associations ont pour but de dénoncer les violations des droits des évangéliques expulsés, de développer des actions en justice et d'exiger la punition des agresseurs. Ce type de démarche a dépassé les frontières confessionnelles et, par une active participation des acteurs évangéliques au sein du Conseil interreligieux du Chiapas qui regroupe

6. "Lo religioso un velo de la lucha interna por el poder en Los Altos ", La Jornada (Mexico), 22 février 1994, p. 16. Une des plus récentes manifestations de violence est le massacre de quarante paysans indiens à Acteal dans le municipe de Chenalho, où plus de la moitié de la population est protestante. Voir La Jornada, 28 mai 1997. 
aussi les Mormons et les Adventistes, elle engage un combat pour la liberté religieuse dans les communautés indiennes. Ces nouveaux acteurs religieux apparaissent ainsi comme de nouveaux intermédiaires capables de mettre en œuvre de nouveaux modèles de relations avec l'État et avec la société indienne. Selon les conjonctures politiques locales, ces nouveaux acteurs s'allient à tel ou tel parti afin de combattre leurs adversaires religieux et politiques qui se trouvent liés à des partis antagoniques. Mais c'est la division religieuse qui détermine les jeux d'alliances et non pas les partis politiques extérieurs qui sont perçus et utilisés comme des recours afin de conforter les positions politiques internes aux communautés définies par la différenciation religieuse. Même si le fractionnement religieux suit des logiques diverses, en particulier liées aux réseaux de parentés, les diverses organisations religieuses non catholiques au sein d'un même municipe présentent un front commun, se retrouvant dans une semblable situation de persécution par le fait même de délégitimer les mécanismes religieux traditionnels de régulation du pouvoir aux mains des caciques locaux. Ceci peut évoluer vers des jeux d'alliances et d'oppositions plus complexes, mais pour l'instant les communautés indiennes apparaissent divisées en deux camps, celui du pouvoir traditionnel lié au catholicisme de la coutume et celui des « rénovateurs » appartenant tous à des sectes évangéliques ou para-chrétiennes et qui peut parfois inclure des catholiques non liés à la religion coutumière. Le déplacement du politique institutionnel vers un politique défini par la scission religieuse a contribué à affaiblir la position des autorités indiennes défendant les mécanismes de contrôle liés à la coutume, en particulier dans la région des Altos de Chiapas. Certains chercheurs considèrent même que dans le municipe de Las Margaritas (région des Cañadas), le conflit religieux peut "repositionner (les évangéliques) comme fer de lance du projet impulsé par les Zapatistes » (Rivera Farfan, 2006 : 356). Afin de comprendre pourquoi les organisations religieuses non catholiques ont pu prendre racine dans les communautés indiennes, il convient d'interroger le type de religiosité qui s’y répand.

\section{Le pentecôtisme ethnique est un rituel}

Les organisations religieuses indiennes non catholiques sont dans leur presque totalité " évangéliques» (protestantes historiques et pentecôtistes) et para-chrétiennes (témoins de Jéhovah, adventistes, mormons). Cependant, les premières sont prédominantes et, parmi elles, le pentecôtisme est le type de religiosité qui les caractérise. Même les églises presbytériennes ou baptistes (qui adoptent l'appellation de "rénovées ») se sont "pentecôtisées » au cours de ces dernières années adoptant la force symbolique d'une ritualité qui parle particulièrement aux pauvres. Le pentecôtisme est fondamentalement un rituel qui sert de langage au groupe, de moyen d'expression effervescent. Le développement rapide de cette expression religieuse dans des sociétés rurales au taux élevé 
d'analphabétisme agit comme un antidote à la violence, au poids des maladies, à l'extrême importance de la sorcellerie au sein d'une population marquée par la marginalité géographique et sociale. On peut relever quatre traits fondamentaux du pentecôtisme.

Il s'agit tout d'abord d'une théologie de l'Esprit, informée par le livre biblique des Actes en son chapitre 2 et liée à un fondamentalisme biblique qui refuse tout rapport cognitif à une tradition religieuse. Pneumatologie et apocalyptique sont deux pôles d'une théologie minimale qui vise à nier l'historicité, à extraire l'acteur religieux des pesanteurs de la réalité sociale coutumière et de l'exclusion. Ce langage abolit la pauvreté. Le biblicisme spiritualisant pentecôtiste ne renvoie pas à la gestion d'un savoir, ni à l'accès savant à un texte. Il renforce un rituel qui permet de gérer l'émotion et de créer un acteur social nouveau dont l'autonomie relative se fonde dans l'acte communautaire dont le langage est la louange. Comme l'a bien vu Corten (1995) pour le pentecôtisme brésilien, la louange est en quelque sorte l'énoncé premier du discours de cet acteur social qu'est toute sociabilité pentecôtiste. Et ce premier énoncé évince tout autre énoncé possible, en particulier celui du contrat (dans la tradition occidentale-libérale). L'énoncé premier du pentecôtisme se traduit dans une séquence rituelle qui inclut un dire spécifique, la glossolalie et un faire qui s'exprime dans des pratiques thaumaturges et exorcistes.

La glossolalie est un discours de pauvres, à la fois extraordinaire et archaïque dans sa forme. Il s'agit « d'un parler en langues » pratiqué collectivement qui se traduit le plus souvent par des formes d'expulsion violente, saccadée et sonore du souffle. Les césures et interjections disparaissent parfois pour se transformer en un cri collectif, une prière brute, montant soudain comme une houle énorme qui s'amplifie par vagues successives et décroît et disparaît soudain. La glossolalie est une grève de la parole régie par les codes de la raison instrumentale et s'inscrit dans la continuité de l'altérité indienne face aux formes dominantes du religieux. C'est un refus de se soumettre au code de la rationalité discursive et de se laisser manipuler par les détenteurs privilégiés de ces codes. C'est une manière de se mettre immédiatement en contact avec le numineux, de se laisser pénétrer par lui, mais aussi de préparer les autres séquences rituelles qui structurent l'action religieuse pentecôtiste, la thaumaturgie et l'exorcisme.

Les rites thaumaturges sont une manière d'opérer des guérisons miraculeuses par imposition des mains ou par des prières en commun. Cette pratique rituelle inscrit le pentecôtisme dans une longue tradition chamanique précolombienne qui met l'accent sur le caractère magique de la guérison. On constate, certes, un abandon apparemment systématique des pratiques religieuses traditionnelles. Mais si les esprits sont diabolisés lors de la conversion, leur efficacité n'est pas niée : les forces spirituelles de la secte sont simplement tenues pour plus puissantes. C'est le cas même chez les témoins de Jéhovah du hameau de Las Ceibas 
pour lesquels « Jéhovah protège ses fidèles de l'influence négative des naguales » (mauvais esprits) (Hernández Castillo, 2001 : 131). On passe au pentecôtisme ou aux cultes para-chrétiens en y retrouvant ce que l'on avait investi dans le système religieux traditionnel. La maladie $\mathrm{y}$ a le même statut. Elle représente un moment de désorganisation des éléments qui composent la personnalité. Elle est comme un message à déchiffrer, car la maladie est, pour le pentecôtiste, le signe d'une malédiction.

Les rites exorcistes permettent d'expulser les démons. Les nombreux pasteurs sont investis des moyens et pouvoirs pour lutter contre les démons. La transe religieuse, la possession, s'inscrit dans les registres des mentalités religieuses populaires. Le dispositif des esprits est disqualifié, mais il est nommé, reconnu, maintenu et même renforcé comme dispositif thérapeutique d'une guerre spirituelle menée par la secte pentecôtiste. Dans cette lutte contre les démons, qui est une constante dans l'activité magique (Cazeneuve, 1972 : 161), le pasteur est proche du chaman. Il est le détenteur d'un pouvoir surnaturel qu'il est parvenu à apprivoiser et qui lui donne la force d'expulser les démons. Dans ce type d'action, le recours à la prière est proche de l'incantation, En effet, le pasteur utilise des formules incantatoires qui ont le pouvoir intrinsèque de faire apparaître le démon. La prière y est aussi présente, mais comme phase ultime qui succède à l'incantation et permet de se concilier le numineux après s'en être emparé.

Essentiellement rituelles, tout comme le catholicisme coutumier, les sociabilités pentecôtistes en milieu ethnique ne se caractérisent pas par la construction d'un rapport cognitif à une tradition religieuse mais par un énoncé premier qui est celui de la louange, expression de l'émotion du pauvre, d'un sentir. Au contraire de la Théologie de la libération qui construisait théoriquement le pauvre " conscientisé » ou du protestantisme historique qui prétendait former des acteurs sociaux autonomes et critiques, le pentecôtisme élabore la catégorie de pauvre, non pas au plan cognitif, mais au plan émotionnel dans une séquence rituelle au travers de laquelle s'atteste la véracité du discours de la secte pentecôtiste en tant que louange. Comme l'explique Corten (1995: 147) pour le contexte brésilien, «dans la louange, le pauvre se met dans le halo de la gloire de Dieu. Face à cette gloire si grande, les inégalités paraissent si petites d'autant plus qu'est convoqué l'univers entier pour louer le Seigneur ». Dans les communautés ethniques du Chiapas, la louange est à la fois un moyen d'invalider la pauvreté et de se libérer des mécanismes de contrôle rituel exercé par le catholicisme coutumier. C'est une libération dont l'intentionnalité est double : élaborer un rituel sur la souffrance pour ceux qui savent qu'ils ne peuvent en sortir et construire un sujet ethnique autonome. Le discours du pentecôtisme n'est donc pas démobilisateur; au contraire, la multiplication de communautés religieuses pentecôtistes correspond à une mobilisation concurrentielle des secteurs dominés des ethnies au travers d'une religiosité qui met en œuvre une séquence rituelle incluant la force du rite, la fascination pour la magie et le recours à la prière. 
Une étude plus fine devrait aussi pouvoir montrer dans le cas des organisations religieuses para-chrétiennes, adventistes, mormones, et témoins de Jéhovah, la manière dont les Indiens redéfinissent les pratiques et leur contenu en fonction de leur univers ancestral de référence.

\section{Protestantisme et ethnicité}

Une abondante littérature anthropologique de combat a dénoncé les sectes, protestantes ou non, pour leur action aliénante au sein des communautés indiennes. On peut se demander pourquoi elles seraient plus un facteur d'acculturation que ne l'a été le catholicisme baroque ou ne l'est le catholicisme actuel qui est souvent polarisé entre partisans de la Théologie de la libération et agents de l'Action catholique. Le catholicisme institutionnel se développe en général sous la conduite d'agents, blancs ou métis, extérieurs aux communautés indiennes, souvent même avec des clercs étrangers. Or les sectes pentecôtistes et le catholicisme de la coutume qui s'affrontent violemment (comme c'est le cas dans le contexte chamula), ont un point commun : tous deux sont des expressions religieuses élaborées par les Indiens eux-mêmes. Le catholicisme coutumier est daté historiquement puisqu'il se constitue tardivement, au XIX siècle, face à l'action redoublée de l'État libéral contre les corporations indiennes (Wasserstrom, $1983: 145-150$ ). Les nouveaux cultes surgissent beaucoup plus récemment, mais aussi en affinité élective avec le chamanisme ancestral. Millénarisme pentecôtiste, thaumaturgie, exorcisme et glossolalie s'inscrivent en continuité avec l'univers religieux indien. Tout comme le catholicisme coutumier, la nouvelle religion repose sur un corps de spécialistes indiens du religieux, les pasteurs et les évangélistes, souvent d'anciens chamans, créateurs de sociabilités religieuses ethniques. Même lorsque les sociétés religieuses non catholiques s'insèrent dans des structures nationales ou internationales, il semble que leur autonomie locale ou régionale soit maintenue tout en pouvant bénéficier de supports et de moyens extérieurs à la région. Ainsi, l'Église nationale presbytérienne du Mexique compte sept « consistoires » dans l'État du Chiapas, dont quatre sont indiens et portent le nom de «tzeltals », «tzotzil » et «chol », soulignant les appartenances ethniques des fidèles et l'enracinement ethnique de l'organisation religieuse. De même, les sociétés religieuses baptistes, de type congrégationaliste, accordent l'autonomie administrative à la sociabilité religieuse locale, respectant ainsi l'idiosyncrasie communautaire. En même temps, ces mouvements religieux mettent l'accent sur le bilinguisme, traduisant les évangiles dans les langues indiennes qui ont été codifiées de cette manière-là pour la première fois. Or, dans un contexte où prédomine l'analphabétisme, l'accès à la maîtrise d'un texte sacré exerce une attraction certaine, d'autant plus qu'il renforce le marqueur linguistique ethnique. Comme le remarque Hernández Castillo (2001 : 176), le simple fait que le mam soit devenu la langue du culte alors qu'il n'était plus autorisé dans les espaces publics, a conduit la population presbytérienne 
mam à participer très activement aux programmes de «sauvetage culturel » promu par les instances indigénistes. De même, ces Églises sont souvent porteuses de petits projets de développement à échelle locale (Cantón Delgado, 2007) qui, même lorsqu'ils échouent, paraissent permettre d'échapper ou de sortir des mécanismes de cooptation mis en place par l'État. Dans des populations où prédominent la pauvreté, l'analphabétisme et les maladies endémiques, les nouveaux mouvements religieux contribuent au développement communautaire comme l'a montré Turner (1979).

Dans ce sens, ils présentent un avantage par rapport au catholicisme coutumier. Ils sont porteurs d'une modernité relative tout en s'ancrant dans l'imaginaire indien. Alors que la " coutume " maintient la communauté sous la double tutelle des caciques et des initiatives étatiques, les nouveaux mouvements religieux semblent asseoir une indépendance possible tout en s'ancrant dans une religiosité rituelle. Ils peuvent renforcer l'autonomie indienne en fondant un nouveau lien communautaire comme Rappaport (1984) l'a montré chez les indiens Paez et Gambianos du sud de la Colombie. Ceci transparaît aussi au Chiapas, dans les villages créés de toute pièce par les migrants des années 1970 et 1980 dans la région des Cañadas et dans les colonies de peuplement périphériques de la ville de San Cristobal. Là, ces communautés pionnières ont pris des noms bibliques (Nueva Jerusalen, Jérico, Betania, Nueva Galilea, Palestina) qui prétendent signifier l'utopie de la reconstruction ethnique, tout en assumant une différence religieuse constitutive.

Ailleurs, dans la zone Fronteriza (Mariscal), les nouveaux mouvements religieux ne semblent pas dissoudre l'identité ethnique, mais plutôt en épouser le contour. Hernández Castillo (1994) constate qu'en pays mam, le presbytérianisme commença, dès les années 1930, par devenir une composante de l'ethnicité. Tous les protestants étaient des Indiens alors que les non-Indiens identifiaient le protestantisme à l'ethnicité (Hernandez Castillo, 1994 : 94). En particulier, le presbytérianisme contribua à la préservation de la langue mam, tout en facilitant la réélaboration de la religion populaire. Face à l'échec des politiques gouvernementales d'intégration, certaines communautés mams ont marqué de manière encore plus abrupte leur différence en se faisant témoins de Jéhovah. Il ne s'agissait plus seulement de s'adapter au changement comme c'était le cas avec le presbytérianisme, mais "de s'en isoler afin de s'en protéger " (Ibid.: 97). Ce faisant, les témoins de Jéhovah du hameau de Las Ceibas "ont développé en général une résistance et un rejet symbolique des institutions étatiques au travers d'un discours messianique critique de la situation actuelle et par la restructuration de la vie communautaire selon leurs propres normes » (Hernández Castillo, $2001: 131)$.

Les relations avec des organisations religieuses internationales engagent, certes, des situations qui peuvent varier en fonction du poids des agents extérieurs. Mais 
il est probable que l'adoption de modèles religieux recomposables comme l'est le pentecôtisme réponde à une longue tradition de résistance et d'adaptation de la part de ces communautés ethniques. Comme le soulignait Gros (1992 : 129), " devenir protestant dans un pays catholique n'est pas abandonner sa "différence", mais en quelque sorte la signifier tout en modernisant sa forme".

Cette continuité de la différence apparaît en ce qui concerne le recours à la langue. Dans le cas des Mams de San Juan Ostuncalco (dans le Guatemala voisin), presbytériens depuis les années 1930, Scotchmer (1991) a montré que ceux-ci ont accru l'usage du mam dans leurs rituels au cours des années 1980 alors que, au même moment, l'espagnol se généralisait dans les échanges journaliers. Ce recours au mam ne s'élabore pas en rupture avec la cosmologie indienne puisqu'il en reprend nombre d'éléments. C'est ce qui explique le grand attrait pour le pentecôtisme qui, par la souplesse de sa doctrine, facilite, encore plus que le presbytérianisme, une telle recomposition. La thèse de Scotchmer permet de constater que, tout comme le catholicisme espagnol d'antan, les nouveaux mouvements religieux " protestants » sont une substance malléable pour les Mayas, en dépit des intérêts exogènes qu'une telle diffusion suppose. L'existence des groupes ethniques dépend de l'entretien de leurs frontières physiques et symboliques. Les mouvements religieux protestants sont, tout autant que le catholicisme coutumier, capables d'articuler la différence ethnique à la variable culturelle et religieuse importée parce qu'elle est reformulée rituellement. Toute l'histoire indienne témoigne en effet d'une formidable capacité d'adaptation au changement. Par un processus d'appropriation-expropriation, les indiens ont prouvé qu'ils peuvent maintenir leur spécificité et "indianiser » les éléments externes qu'on leur imposait. Le catholicisme de la coutume fournit un bon exemple d'une acculturation " autogérée » de ces éléments culturels apparemment assimilés, mais détournés et investis par des significations propres. Il en va de même dans le cas des nouveaux mouvements religieux informés par le pentecôtisme ou d'autres traditions religieuses para-chétiennes.

\section{Conclusion}

Un nouveau paysage religieux s'est dessiné en terre indienne maya du Chiapas. Il prend la forme du conflit des appartenances religieuses suscitées par des groupes d'intérêts opposés. Les frontières du politique ne passent plus uniquement par les appareils partisans imposés par les agents extérieurs aux communautés indiennes. Une nouvelle géographie sacrale du politique s'est structurée qui déplace les intérêts liés aux partis politiques vers ceux structurés autour des appartenances religieuses. Il y a dans ce processus la remise en question radicale de la légitimité du politique tel qu'il est défini par l'État au détriment des autonomies ethniques. Au lieu d'accompagner un processus de sécularisation du politique, la guerre confessionnelle déplace le politique dans un espace sacral qui reste sous contrôle 
des acteurs indigènes par le biais de nouvelles ritualités, indiennes elles aussi. Les nouvelles ritualités pentecôtistes ne sont pas moins indiennes que celles, coutumières, liées au catholicisme baroque de dévotion. Elles servent à construire une ethnicité plurielle au plan religieux et par conséquent politico-religieux dans des sociétés non sécularisées.

En même temps, s'engage un processus de pluralisation des croyances qui n'est pas un signe de sécularisation des sociétés indiennes. Certes, l'adhésion aux organisations protestantes, pentecôtistes ou autres, semble impliquer à la fois un refus et une apparente désacralisation du temps imposé par le rythme du calendrier des fêtes rituelles catholiques. Ceci peut conduire à une certaine rationalisation des croyances et de la vie économique. Cependant, contre toute rationalisation théologique, le recours au merveilleux magique subsiste au travers de l'effervescence pentecôtisante caractéristique de la plupart des nouveaux mouvements religieux. Ceux-ci, bien que brisant l'homogénéité religieuse ethnique, reconstruisent un lien social, ethnique lui aussi parce que fondé dans une ritualité religieuse en continuité avec l'imaginaire ancestral. Il en va ainsi aussi bien dans les municipes indiens que dans les laboratoires de peuplement pionniers de la frontière lacandonne ou au sein des microcommunautés religieuses aux noms bibliques des périphéries de San Cristobal. L'ethnicité n'est pas un fait immuable, " elle implique toujours un processus de sélection de traits culturels dont les acteurs se saisissent pour en faire des critères d'assignation ou d'identification " (Poutignat et alii, 1995 : 141). Les nouveaux mouvements religieux, protestants ou non, tout autant que le catholicisme remplissent cette fonction emblématique et servent de nouveaux marqueurs d'appartenance. C'est pourquoi, même après plus de dix ans d'expulsion de leurs communautés chamulas ou zinacantèques, les indiens « protestants » cherchent encore à retourner sur leur territoire ${ }^{7}$. Mais, afin de ne pas se voir contraints à signer des "arrangements " leur intimant l'ordre d'accepter le retour au statu quo, c'est-à-dire la réintégration dans la ritualité politico-religieuse coutumière sanctionnée par le politique légitimé par l'appareil d'État, ils s'appuient sur des comités régionaux, nationaux et même internationaux de défense des droits de l'homme afin de demander le respect de leur différence rituelle et par conséquent politique dans le sens non partisan du terme. Le processus de pluralisation religieuse entraine la fin du monolithisme religieux de la coutume et facilite le recours à des médiations extérieures à l'ethnie, mais n'implique pas l'assimilation dans la société globale. En revendiquant le droit à leur réintégration, les Indiens dissidents refusent la violence symbolique et physique imposée par les caciques et affirment que leur identité passe par le maintien privilégié du rapport à l'espace ethnique et à la langue, précisément parce que l'ethnicité est recomposée dans ces communautés imaginaires que sont

7. «Retornan indígenas evangélicos, tras 10 años, a Zinacantan », La Jornada (Mexico), 30 mai 1995, p. 17. Les expulsés chamulas des périphéries de San Cristobal luttent aussi pour regagner leur territoire ethnique. À ce sujet voir El Universal (Mexico), $1^{\text {er }}$ mars 1994, pp. 1, 10. 
les sectes. Dans cette lutte et dans cette rivalité rituelle se joue un déplacement du politique comme tentative d'éradiquer la violence intra-ethnique légitimée à la fois par la religion coutumière et les partis au pouvoir.

Jean-Pierre BASTIAN

Université Marc Bloch (Strasbourg) - Centre de Sociologie des religions

et d'éthique sociale

bastian@umb.u-strasbg.fr

Aramoni Calderon Dolores, 1992, Los refugios del sagrado, religiosidad, conflicto y resistencia entre los zoques de Chiapas, Mexico, Conaculta.

AUBRY André, 1988, Les Tzotzils par eux-mêmes, récits et écrits de paysans indiens $d u$ Mexique, Paris, l'Harmattan.

Barabas Alicia M., 1989, Utopias indias, movimientos socioreligiosos en México, Mexico, Grijalbo.

BASTIAN Jean-Pierre, 1997, La mutación religiosa de América Latina. Para una sociología del cambio social en la modernidad periférica, Mexico, Fondo de Cultura Económica.

Cancian Frank, 1965, Economics and Prestige in a Maya Community. The Religious Cargo System of Zinacantan, Standford, Standford University Press.

-, 1992, The Decline of the Community in Zinacantan: Economy, Public Life and the Social Stratification, 1960-1987, Standford, Standford University Press.

Canton Delgado Manuela, 1994, "Protestantismo y violencia en tierras mayas : los casos de Guatemala y Chiapas (Mexico) ", Antropologia (Madrid), 8, pp. 31-58.

-, 2007, «Filiations protestantes, habitudes économiques et développement. Une perspective anthropologique à partir du cas guatémaltèque ", in Jean-Pierre BASTIAN, (dir.), Religions, valeurs et développement dans les Amériques, Paris, L’Harmattan, pp. 137170.

Cazeneuve Jean, 1972, Sociologie du rite, Paris, Presses Universitaires de France.

COlLIER Georges A., 1994, "The Rebellion in Chiapas and the Legacy of Energy Development », Mexican Studies/Estudios Mexicanos, (San Diego), 10/2, pp. 371-382.

CORTEN André, 1995, Le pentecôtisme au Brésil. Émotion du pauvre et romantisme théologique, Paris, Karthala.

Dehouve Danièle, 2006, Essai sur la royauté sacrée en République mexicaine, Paris, CNRS-Éditions.

Dow James W., 1993, «Protestantismo en el campo : causas materiales del abandono de fiestas en la sierra oriente de Hidalgo ", Notas Americanas (Cholula, Mexico), pp. 123-130.

FAVRE Henri, 1971, Changement et continuité chez les Mayas du Mexique, Paris, Anthropos.

GABBERT Wolfgang, 1999, «Violence and Social Change in Highland Maya Communities, Chiapas, Mexico ", Ibero-Amerikanisches Archiv, Berlin, 25/3-4, pp. 351-374.

GARCIA Ruiz Jesús, 1992, "De la identidad aceptada a la identidad elegida ; el papel de lo religioso en la politización de las identificaciones etnicas en Guatemala ", Estudios Sociológicos (Mexico), X/30, pp. 713-733. 
Gros Christian, 1992, "Fondamentalisme protestant et populations indiennes : quelques hypothèses ", Cahiers des Amériques Latines, 13, pp. 119-134.

Hernandez Castillo Rosalva Aída, 1994, "Identidades colectivas en los márgenes de la nación : etnicidad y cambio religioso entre los mames de Chiapas ", Nueva Antropología, Revista de Ciencias Sociales (Mexico), XIII/45, pp. 83-106.

-, 2001, La otra frontera. Identidades multiples en el Chiapas poscolonial, Mexico, CIESAS.

INEGI, XI et XII censo general de poblacion, 1991, 2001, « Chiapas, resultados definitivos », México, Instituto Nacional de Estadística, Geografía e Informática, pp. 215-220.

Lisbona Guillen Miguel, 1991, "Religion en Ocotepec, Chiapas », Anuario del Instituto chiapaneco de cultura, pp. 37-74.

Lundius Jan, 1993, "Rural Cofradias: A Comparative Study of Syncretism, Fertilility Beliefs and Communal Worship among Peasants in Medieval Europe, the Dominican Republic and Mayan Regions of Central America ", Ibero Americana, Nordic Journal of Latin American Studies (Stockholm), XXIII/1-2, pp. 39-88.

Poutignat Philippe, Streiff-Fenart Jocelyne, 1995, Théories de l'ethnicité, Paris, Presses Universitaires de France.

RAPPAPORT Joanne, 1984, "Las misiones protestantes y la resistencia indígena en el sur de Colombia ", América Indígena (Mexico), XLIV/1, pp. 111-126.

Rivera Farfan Carolina et Lisbona Guillen Miguel, 1992, "La organisación religiosa de los zoques. Problemas y lineas de investigación ", Anuario del Instituto chiapaneco de cultura, pp. 70-103.

Rivera FARFAn Carolina et alii, 2005, Diversidad religiosa y conflicto en Chiapas. Intereses, utopias y realidades, Mexico, UNAM.

Scotchmer David G., 1989, "Symbols of Salvation. A local Mayan Protestant Theology ", Missiology. An International Review, 17/3, pp. 293-310.

-, 1991, Symbols of Salvation: Interpreting Highland Maya Protestantism in Context, Ph.D. Dissertation, Albany, State University of New York.

SiverTs Henning, 1969, Oxchuc, Mexico, Instituto Indigenista Interamericano.

SMith Waldemar R., 1981, El sistema de fiestas y el cambio económico, Mexico, Fondo de Cultura Económica.

TejerA Hector, 1991, «Vivir para creer, Chiapas, política y religion », México Indígena (Mexico), pp. 19-22.

Turner Paul R., 1979, «Religious Conversion and Community Development ", Journal for the Scientific Study of Religion, 18-3, pp. 252-260.

Watanaba John M., 1995, "Unimagining the Maya: Anthropologist, Others and the Inescapable Hubris of Authority ", Bulletin of Latin American Research, 14/1, pp. 25-45.

WASSERSTROM Robert, 1983, Class and Society in Central Chiapas, Berkeley, University of California Press. 


\section{Résumé}

La différenciation religieuse accélérée que vivent les communautés indiennes dans l'État du Chiapas, au Mexique, est le fruit de la violence intra-ethnique. Dans ce mouvement de changement des appartenances religieuses, se définissent de nouveaux langages religieux et de nouveaux modes de communication des identités individuelles et collectives qui passent par la mise en forme de séquences rituelles structurées en grande partie par le pentecôtisme. Au travers de l'adoption de ces nouvelles croyances et pratiques religieuses flexibles se joue à la fois l'ancrage dans les traditions religieuses ancestrales et la construction d'une modernité indienne fondée sur le droit et la reconnaissance du pluralisme idéologique. Ceci s'opère sans détruire la différence ethnique mais plutôt en recomposant ses éléments dans le sens d'une modernité endogène relativement autonome. La pluralisation religieuse donne l'opportunité de construire une définition religieuse du politique qui échappe aux régulations exogènes du politique légitime imposées par l'État tout en s'appuyant sur les principes juridiques de la liberté de culte. L'adoption de nouvelles expressions religieuses non catholiques continue de souligner la différence avec le reste de la société mexicaine et permet de chercher les moyens de combattre les inégalités sociales au sein des sociétés ethniques tout autant que le rapport de subordination de l'ethnie à la nation.

Mots-clés: Mexique, Chiapas, religion, violence, politique.

\section{Abstract}

Intra-ethnic violence is fueling rapid religious differentiation in the state of Chiapas (Mexico). Changes in religious affiliation produce new religious codes and modes of communicating individual and collective identities heavily influenced by ritual sequences borrowed from the Pentecostal tradition. At stake in the implementation of these new beliefs and practices are the reaffirmation of ancestral religious traditions and the possibility of shaping a Mayan modernity that recognizes ideological pluralism. Rather than denying the ethnic specificity of Mayan society, this process adapts elements of ethnic identity to meet the needs of a relatively autonomous and endogenous modernity. Religious pluralization provides the Maya people with the possibility of defining politics in a religious way, allowing them to assert their independence from the central government and its exogenous political regulation while appealing to the legal principles of religious freedom. Not only does the adoption of new, non-Catholic expressions of religiosity underline the distinctiveness of the Mayan civilization within Mexican society, it opens up new perspectives in the struggle against endogenous social inequalities and in the fight against the domination of the nation-state.

Key words: Mexico, Chiapas, religion, violence, politics.

\section{Resumen}

Las diferenciaciones religiosas acceleradas que experimentan las comunidades indígenas del estado de Chiapas son el fruto de la violencia intraétnica. En este movimiento de cambio de las afiliaciones religiosas, se definen nuevos lenguajes religiosos y modos de comunicación de las identidades individuales y colectivas que pasan por la elaboración de nuevas formas de secuencias rituales estructuradas en gran parte 
por el pentecostalismo. Por la adopción de estas nuevas creencias y prácticas religiosas fléxibles se esta redefiniendo el lazo con las tradiciones religiosas ancestrales y se construye une modernidad indígena fundada en el derecho al pluralismo ideológico. Esto se produce sin destruir la diferencia étnica y recompone elementos de aquella en el sentido de una modernidad endógena relativamente autónoma. La pluralización religiosa ofrece la oportunidad de construir une definición religiosa endógena de lo político en continuidad con el imaginario maya y lo hace escapar a las regulaciones exógenas de lo político official impuesto por el estado nacional, sin prescindir del derecho constitucional a la libertad religiosa. La adopción de nuevas expresiones religiosas no católicas sigue reforzando la diferencia étnica y permite encontrar mediaciones para combatir las desigualdades sociales en el seno mismo de las sociedades étnicas e intentar modificar las relaciones de subordinación de la etnia a la nación.

Palabras clave: Mexico, Chiapas, religiones, violencia, politica. 
